\title{
Natural Selection, Infectious Transfer and the Existence Conditions for Bacterial Plasmids
}

\author{
Carl T. Bergstrom, Marc Lipsitch ${ }^{1}$ and Bruce R. Levin \\ Department of Biology, Emory University, Atlanta, Georgia 30322 \\ Manuscript received October 8,1999 \\ Accepted for publication April 20, 2000
}

\begin{abstract}
ABST RACT
Despite the near-ubiquity of plasmids in bacterial populations and the profound contribution of infectious gene transfer to the adaptation and evolution of bacteria, the mechanisms responsible for the maintenance of plasmids in bacterial populations are poorly understood. In this article, we address the question of how plasmids manage to persist over evolutionary time. Empirical studies suggest that plasmids are not infectiously transmitted at a rate high enough to be maintained as genetic parasites. In part i, we present a general mathematical proof that if this is the case, then plasmids will not be able to persist indefinitely solely by carrying genes that are beneficial or sometimes beneficial to their host bacteria. Instead, such genes should, in the long run, be incorporated into the bacterial chromosome. If the mobility of host-adaptive genes imposes a cost, that mobility will eventually be lost. In part ii, we illustrate a pair of mechanisms by which plasmids can be maintained indefinitely even when their rates of transmission are too low for them to be genetic parasites. First, plasmids may persist because they can transfer locally adapted genes to newly arriving strains bearing evolutionary innovations, and thereby preserve the local adaptations in the face of background selective sweeps. Second, plasmids may persist because of their ability to shuttle intermittently favored genes back and forth between various (noncompeting) bacterial strains, ecotypes, or even species.
\end{abstract}

M

O ST bacteria from natural sources abound with a variety of semiautonomous genetic molecules that are transmitted vertically, in the course of cell division, and horizontally by infectious transfer. Plasmids - a particularly prominent class of these accessory genetic elements- either code for their own infectious transfer, usually by conjugation and more rarely as viruses, or have specific mechanisms (e.g., mobilization transfer) that facilitate their infectious transmission by hitchhiking with self-transmissible plasmids (Summer s 1996). While plasmids may be best known as tools of molecular biologists and biotechnologists, or as bearers of problematic genes for virulence and drug resistance, they provoke a number of intriguing ecological and evolutionary questions that have only started to be addressed (Levin and Bergst rom 2000). The most elemental of these questions for extant plasmids is what Campbel I (1961) referred to as "existence conditions." Under what conditions can these elements become established and be maintained in bacterial populations?

While the fitness burden imposed on the host bacteria by the carriage and replication of plasmids may be small, it must be nonzero. And, although there are mecha-

Corresponding author: Carl T. Bergstrom, Department of Biology, Emory University, 1510 Clifton Rd., Atlanta, GA 30322.

E-mail: cbergst@emory.edu

${ }^{1}$ Present address: Department of Epidemiology, $\mathrm{H}$ arvard School of Public Health, 677 Huntington Ave., Boston, MA 02115. nisms to reduce the rates at which plasmids are lost in the course of cell division (Nordst röm et al. 1984; Gerdes et al. 1986; Luria and Suit 1987; Nordström and Aust in 1989; Mongold 1992; Paulsson and Ehrenber $g$ 1998), the rates of loss due to vegetative segregation remain strictly positive. In the absence of some mechanism countering their intrinsic costs and segregation loss rates, plasmids would eventually be removed from bacterial populations. Therefore, plasmids' persistence requires one or both of two basic mechanisms: infectious transmission and maintenance as genetic parasites or selection on hosts for the genes that the plasmids carry. Let us briefly consider these in turn.

Plasmids ( particularly those that code for their own transmission) can transfer copies of themselves to plasmid-free bacteria at seemingly high rates. In theory it would be possible for plasmids to persist as genetic parasites; their horizontal transmission rates could in principle overcome both the fitness costs associated with their carriage and the losses due to vegetative segregation. If this were so, then these elements could be maintained by infectious tranfer even when they engender a substantial cost to the fitness of their host bacteria. On the other hand, the conditions for this to obtain are relatively restrictive, and particularly so for plasmids that are not self-transmissible (St ew art and Levin 1977; Levin and Rice 1980). On the basis of primarily in vitro estimates of plasmid transfer rates, fitness costs, and segregation rates, and densities and physiological conditions of natural populations (Levin et al. 1979; Levin 
1980; Simonsen et al. 1990; Simonsen 1991; Gordon 1992), it has been postulated that, in practice, these elements cannot be maintained as genetic parasites (Levin 1993; but also see Lundquist and Levin 1986). If this is correct, the obvious question arises: How are plasmids maintained?

The alternative possibility is that plasmids could persist by bearing (or hitchhiking alongside) genes that increase the fitnesses of their bacterial hosts. Several hypotheses have been proposed along these lines, often variations on a theme of plasmids "delivering" occasionally useful genes to bacteria that need them. E ber $h$ ar $d$ (1990) suggests that plasmids carry genes for exotic functions needed only in a small subset of habitats or on rare occasions. When favored, they can spread by conjugation; when disfavored, they do not impose their costs on the entire bacterial population. Summer s (1996) likens the wide array of genes carried on plasmids to a genetic "lending library," arguing that plasmids are useful because they need not be maintained in every individual in the population. Though appealing as an analogy, this is not intended as a mechanistic explanation. Turner et al. (1998) propose a somewhat different mechanism: perhaps plasmids persist by transferring onto immigrant strains that are sweeping through the population, hitchhiking to high frequency on each selective sweep. H orizontal transfer, they argue, "may be seen as an adaptation that allows a parasite [i.e., the plasmid] to move onto superior hosts that emerge."

Certainly, there can be intense selection for one or more plasmid-borne genes, as is the case during antibiotic treatment when resistance is plasmid-encoded. Under such conditions, bacteria carrying those plasmids would be at a considerable advantage relative to bacteria without them. We note, however, that few if any of the genes borne by bacterial plasmids are under strong positive selection at all times. Moreover, the simple presence of selectively advantageo us genes on bacterial plasmids is not sufficient to explain plasmid maintenance. Genes originally carried on the plasmid presumably can be sequestered by the chromosome; the host bacterium could then continue to express these genes but dispense with the plasmid and its associated costs. (H ere we are assuming that the fitness effects of the selected genes are the same whether they are plasmid-borne or chromosomally encoded. No additional advantage is assumed to accrue from factors such as the increased copy number associated with the plasmid-borne lifestyle.) Consequently, one not only needs to compare plasmidbearing cells with plasmid-free cells, but also to compare plasmid-bearing cells with plasmid-free cells that have incorporated some or all of the plasmid-encoded hostbeneficial genes into the chromosomal genome.

How, then, do plasmids manage to persist over evolutionary time? How have these elements evolved and maintained the seemingly costly machinery (Ippen-
IhI er 1989; Summer s 1996) necessary for their infectious transmission? Why do they engage in infectious (horizontal) transmission at all? We attempt to answer these questions quantitatively in the analysis that follows.

0 verview: In this article, we use mathematical models and computer simulations to explore the existence conditions of plasmids. The most reasonable interpretation of the available evidence is that plasmids cannot be maintained solely by horizontal transfer in single populations. We therefore explore other processes that can maintain plasmids.

In part $\mathrm{i}$, we show that plasmids cannot persist simply by bearing genes that are beneficial to their bacterial hosts. To do so, we determine the conditions under which natural selection will favor plasmidsbearing beneficial or occasionally beneficial genes. We demonstrate that under these conditions, the plasmid-encoded genes will ultimately be sequestered by the host chromosome and the plasmid will be unable to persist. Thus the very conditions that allow plasmid-bearing cells to persist in competition with plasmid-free cells will eventually select for incorporation of the plasmid-borne genes into the bacterial chromosome and subsequent loss of the plasmid. In perusing this section, some readers may wish to skim the mathematical details and focus primarily on the statements of results.

In part $\mathrm{ii}$, we present a pair of simple illustrative models intended to highlight two processes that could account for the long-term existence of bacterial plasmids. First, we treat the role played by plasmids when selective sweeps are occurring at chromosomal loci. Second, we examine the way in which plasmids may persist by carrying genes across clone, ecotype, or species boundaries.

\section{PART I: MECHANISMS THAT CANNOT MAINTAIN PLASMIDS}

The basic model and assumptions: In the following analysis, we employ a generalized version of St ewart and Levin's (1977) model of plasmid population dynamics in a single-clone population of bacteria. The model compares the growth rates of two kinds of bacterial populations, those with and those without the plasmid, with densitiesand designations $\mathrm{P}(\mathrm{t})$ and $\mathrm{F}(\mathrm{t})$ bacterial cells per milliliter, respectively, at time t. The growth rate per hour of plasmid-free cells at time $t$ is given by $\psi(t) \geq 0$, and the washout, death, or loss rate per hour at $t$ is given by $u(t) \geq 0$. Plasmid-bearing cells grow at a slower rate than plasmid-free cells, due to the energetic and other costs associated with plasmid carriage, replication, and gene expression: the growth rate of plasmid-bearing cells is $\psi(t)(1-\alpha)$, where $\alpha \geq 0$ represents the "cost" of plasmid carriage expressed as a fractional reduction in replication rate. Conjugation occurs via a mass action process at a rate proportional to the product of the densities of plasmid-bearing and plasmid- 
free cells and a rate parameter $\gamma(\mathrm{t}) \geq 0$ (milliliters per cell per hour; St ewart and Levin 1977; Simonsen et al. 1990). Plasmid loss via segregation occurs at rate $\tau(t) \geq 0 / \mathrm{hr}$. Finally, we assume there is an upper bound on the maximum attainable bacterial density of $\mathrm{N}^{+}$ cells/ $\mathrm{ml}$ and note that $\mathrm{N}^{+} \geq \max _{\mathrm{t}}(\mathrm{F}(\mathrm{t})+\mathrm{P}(\mathrm{t})$ ).

As explained above, we make the assumption that plasmids cannot persist as purely parasitic genetic elements.

Assumpt ion 1. Plasmids impose nonzero fitness costs on their host bacteria and suffer nonzero rates of loss by vegetative segregation. Even at the maximum host density, plasmids cannot transfer at a sufficient rate to overcome these costs and therefore cannot be maintained as parasites. Instead, plasmids can be maintained only with the assistance of selection favoring the bacteria that carry them.

We can express this assumption in the mathematical terms of our model. With the definitions above, the rates of change of plasmid-bearing and plasmid-free cell densities at time $t$ are

$$
\begin{aligned}
& \dot{P}(\mathrm{t})=\mathrm{P}(\mathrm{t})(\psi(\mathrm{t})(1-\alpha)-\tau(\mathrm{t})+\gamma(\mathrm{t}) \mathrm{F}(\mathrm{t})-\mathrm{u}(\mathrm{t})) \\
& \dot{\mathrm{F}}(\mathrm{t})=\mathrm{F}(\mathrm{t})(\psi(\mathrm{t})-\gamma(\mathrm{t}) \mathrm{P}(\mathrm{t})-\mathrm{u}(\mathrm{t})) .
\end{aligned}
$$

By Assumption 1, in the absence of beneficial effects on host fitness, the number of plasmid-bearing cells never grows as quickly as the number of plasmid-free cells; i.e, for all timest,

$$
\frac{\dot{\mathrm{P}}(\mathrm{t})}{\mathrm{P}(\mathrm{t})}<\frac{\dot{\mathrm{F}}(\mathrm{t})}{\mathrm{F}(\mathrm{t})}
$$

Thisimplies that the following inequality, which we label as the Stewart and Levin criterion ( $S \& L$ criterion, for short), holds for all $t$,

$$
\psi(t) \alpha+\tau(t)>N^{+} \gamma(t) .
$$

In words, the rate at which plasmids spread by conjugation is al ways less than the rate of loss due to the combined effects of segregation and selection on the bacterial host. The Stewart and Levin criterion is our desired mathematical formulation of Assumption 1; we take it as given for the remainder of the article. When the $S \& L$ criterion holds, plasmids must do something useful for their hosts in order to be maintained.

This model is more general than previous treatments of plasmid population dynamics for three reasons. In other models, the growth rate $\psi(t)$ is usually treated as a function of the available resource concentration and other parameters. Here we avoid the imposition of any such assumptions and instead express the growth rate as an unspecified function of time to allow ourselves maximal generality. The segregation rate $\tau(t)$ enjoys similar generality. By making the washout rate $u(t)$ an unspecified function of time, we allow the model to cover growth regimes ranging from chemostat growth [in which $u(t)$ would be constant] to serial transfer [in which $u(t)$ would usually be zero and occasionallyduring passages - would be very large].

We did make the usual assumption that conjugation occurs via mass action. This is for the sake of expositional simplicity alone. Strictly speaking, the mass-action form of the conjugation process is not necessary for any of the analyses presented in this article, so long as the total conjugation rate is appropriately bounded to ensure that under the best of conditions the plasmid does not transfer at a rate high enough to overcome its costs of carriage. Similarly, we assume for simplicity that segregation does not generate viable plasmid-free cells, due to postsegregational killing or similar measures. The conditions for plasmid persistence when segregation does generate viable plasmid-free cells are even more restrictive; results parallel to those of this section can be easily derived for that model as well.

Plasmids cannot persist under constant selection: If plasmids were the sole sources of genes that provide a growth-rate advantage to their bacterial hosts, and if these genes could not be incorporated into the host chromosome, then plasmids could indeed persist simply by host-level selection. To demonstrate this, we add a term $\beta$ to the model, representing the fitness consequences to the host of the plasmid-borne genes. Thus, if the growth rate of plasmid-bearing cells is $\psi(t)$ ( 1 $\alpha)(1+\beta)$, then the following condition, if it is true for all times $t$, is sufficient (but not necessary) to ensure plasmid persistence:

$$
\beta>\frac{\tau(\mathrm{t})+\psi(\mathrm{t}) \alpha-\gamma(\mathrm{t})(\mathrm{F}(\mathrm{t})+\mathrm{P}(\mathrm{t}))}{\psi(\mathrm{t})(1-\alpha)} .
$$

O ur first (if not particularly surprising) result follows directly.

Resul $t$ 1. Even in the absence of conjugative transfer, plasmids can invade and be maintained in a bacterial population provided that they carry sufficiently beneficial genes that are not present in the chromosomal genome of the plasmid-free cells.

However, there is no reason to expect that plasmids will remain the sole sources of these genes over evolutionary time. Numerousmechanisms, adaptive or otherwise, facilitate the movement of genes between plasmids and the bacterial chromosome and serve to create chromosomal variants of the plasmid-encoded genes. Plasmid-bearing cells eventually will face competition from plasmid-free cells, which have incorporated the useful (or "focal") plasmid genes directly into their chromosomes, as well as from plasmid-free cells that lack these genes. To explain the long-term evolutionary maintenance of plasmids, we must further extend the model to consider the dynamics of plasmidschallenged by both types of competitors.

Consider competition between plasmid-bearing cells $P$ and plasmid-free cells $F$, as before, and also define $a$ 
third type of cell: plasmid-free cells carrying the focal genes on the chromosome. We refer to these cells as "chromosomals," and represent their density at time $t$ by the variable $C(t)$. There is also a fourth possible type of cell, plasmid-bearing cells that also have the focal genes on the chromosome. These cells will have the same growth rate as the plasmid-bearing cells $P$, even in the best-case scenario in which this duplication of genes carries no additional cost. When segregation rates are reasonably low, these cells can be grouped with the plasmid-bearing cells $P$ without appreciably altering the dynamics. For the sake of mathematical brevity we make this assumption in the analysis that follows, though this is not essential to any of the results derived.

The growth rates of these three types are

$$
\begin{gathered}
\dot{\mathrm{P}}(\mathrm{t})=\mathrm{P}(\mathrm{t})(\psi(\mathrm{t})(1-\alpha)(1+\beta)-\tau(\mathrm{t}) \\
\quad+\gamma(\mathrm{t})(\mathrm{F}(\mathrm{t})+\mathrm{C}(\mathrm{t}))-\mathrm{u}(\mathrm{t})) \\
\dot{\mathrm{F}}(\mathrm{t})=\mathrm{F}(\mathrm{t})(\psi(\mathrm{t})-\gamma(\mathrm{t}) \mathrm{P}(\mathrm{t})-\mathrm{u}(\mathrm{t})) \\
\dot{\mathrm{C}}(\mathrm{t})=\mathrm{C}(\mathrm{t})(\psi(\mathrm{t})(1+\beta)-\gamma(\mathrm{t}) \mathrm{P}(\mathrm{t})-\mathrm{u}(\mathrm{t})) .
\end{gathered}
$$

We can immediately observe that in this system, the condition for plasmid-bearing cells to outgrow chromosomals at any time $t$ is

$$
\gamma(\mathrm{t})(\mathrm{F}(\mathrm{t})+\mathrm{C}(\mathrm{t})+\mathrm{P}(\mathrm{t}))>\psi(\mathrm{t}) \alpha(1+\beta)+\tau(\mathrm{t}) .
$$

If the Stewart and Levin criterion holds, then this will never be the case for a plasmid bearing beneficial ( $\beta>$ 0 ) genes. Our second result then follows, given the $S \& L$ criterion.

Result 2. When the plasmid genes that are useful to their bacterial hosts can be incorporated into the bacterial chromosome, plasmids cannot persist by bearing beneficial genes under constant selection.

Plasmids cannot persist under fluctuating selection: What happens when selective conditions fluctuate? For example, what happens when $\beta$ is replaced by $\beta(\mathrm{t}) \geq$ -1 , a sometimes-negative function of time? Though either chromosomals or plasmid-free cells will be favored at any given time, can plasmids persist over the long-term under fluctuating selection? This might seem plausible; under certain conditions, a genotype can persist under fluctuating selection even if it is never the most-fit type in the population ( $\mathrm{H}$ al dane and Jayakar 1963; Gillespie 1973; Yoshimura and Jansen 1996). In this case, however, we prove that the plasmid inevitably will be out-competed in the long run.

Starting from an initial population with chromosomal density $C_{0}$ and plasmid-free density $F_{0}$, the ratio of plasmid-free cells to chromosomals at time $T$ is

$$
\frac{F(t)}{C(t)}=\frac{F_{0} e^{\int \delta \psi(t) d t}}{C_{0} e^{\int \delta \psi(t)(1+\beta(t)) d t}}=\frac{F_{0}}{C_{0}} e^{-\int \delta \psi(t) \beta(t) d t} .
$$

As time goes to infinity, this will converge to 0 when the expectation of $\psi(t) \beta(t)>0$ and will approach infin- ity when the expectation of $\psi(t) \beta(t)<0$, where the expectation is simply

$$
E[\psi(t) \beta(t)]=\lim _{T \rightarrow \infty} \frac{\int_{0}^{T} \psi(t) \beta(t) d t}{T} .
$$

We take these cases in order. First, suppose that $E[\psi(t) \beta(t)]>0$. By the $S \& L$ criterion,

$$
\begin{array}{r}
E[\psi(t)(1+\beta(t))]>E[\psi(t)(1-\alpha)(1+\beta(t)) \\
\left.-\tau(t)+\gamma(t) N^{+}\right] .
\end{array}
$$

Therefore, the expected growth rate of chromosomal cells (left-hand side) exceeds the expected growth rate of plasmid-bearing cells (right-hand side). The ratio of plasmid-bearing to chromosomal cells at time t will converge to 0 as $t$ becomes large, and plasmid-bearing cells will necessarily be lost.

Second, when $E[\psi(t) \beta(t)]<0$, it follows from the $S \& L$ criterion that

$$
\mathrm{E}[\psi(\mathrm{t})]>\mathrm{E}\left[\psi(\mathrm{t})(1-\alpha)(1+\beta(\mathrm{t}))-\tau(\mathrm{t})+\gamma(\mathrm{t}) \mathrm{N}^{+}\right] .
$$

Here, the expected growth rate of plasmid-free cells (left-hand side) exceeds the expected growth rate of plasmid-bearing cells (right-hand side), and plasmidbearing cells will be lost. Our third result, again based on Assumption 1, follows immediately.

Resul t 3. In a single population subject to constant or fluctuating selection, plasmids cannot persist by bearing beneficial or sometimes-beneficial genes, when these genes can be alternatively incorporated into the chromosomal genome.

To look at this another way, if the plasmid-borne genes are beneficial on average, they will be incorporated into the bacterial chromosome and the plasmids will be discarded. If they are not beneficial on average, they and the plasmids that carry them will be discarded.

Thus far, we have assumed that the fractional cost of plasmid carriage $\alpha$ does not change with time. If we relax this assumption, Result 3 need not hold. Because we consider this a formal possibility of the model rather than a likely explanation of plasmid persistence, treatment of this scenario has been relegated to appendix $a$.

Plasmids cannot persist in a metapopulation: When-as suggested by empirical results ( L evin et al. 1979) - conjugation and segregation occur at a rate proportional to growth, and plasmid-borne and chromosomal versions of a gene do not differ in their rates of migration, Result 3 generalizes to a model with multiple habitats (the proof is outlined in appendix b).

Resul t 4. In a metapopulation composed of multiple bacterial habitats, each subject to correlated or uncorrelated fluctuations in selective conditions, plasmids cannot persist by bearing selected genes, given that (1) plasmid-encoded genes can alternatively be incorporated into the chromosomal genome, (2) conjugation 
and segregation occur at rates proportional to bacterial growth, and (3) plasmid-encoded genes do not directly affect the rates of bacterial movement between habitats.

At equilibrium, we do not expect to observe plasmids simply because they code for useful or sometimes-useful genes. This leaves us with the problem of explaining the prevalence of plasmids in natural populations. At least three possibilities exist, one of which seems extremely unlikely, and two of which merit more serious consideration.

First, the unlikely possibility: any particular lineage of plasmids is in effect a genetic accident, doomed to eventual extinction. If so, plasmids are observed at the present time because the system has not yet reached equilibrium. Taking this idea further, if plasmids are continually being generated de novo and plasmid loss takes sufficiently long, a "creation-loss" balance could be maintained in which plasmids are always present at a reasonable frequency. We believe that this possibility can be dismissed, for it seems improbable that the longstanding sequence divergence among plasmids, and the highly derived plasmid adaptations for conjugation and for the avoidance of segregation loss, are the consequences of genetic accidents rather than the products of active selection.

The models considered thus far leave open two possibilities that seem to us to be more reasonable. Both involve the realities of bacterial population structure, e.g., population subdivision, local adaptation, and periodic selection (Maynard Smit h 1991; Cohan 1994b), that were not incorporated into the simple "single population" model above. One such possibility is that plasmids persist through their ability to transfer into bacterial strains sweeping through the population. The other ( not mutually exclusive) possibility is that plasmids persist because of their ability to transfer across ecotype and species boundaries. We consider these two explanations in part ii of this report.

\section{PART II: WHEN PLASMIDS CAN}

...it is the policy of the state of $\mathrm{New}$ M exico to enhance the selfesteem of students in the classroom, yet the teaching of a theory that indicates that children evolved in a meaningless manner through highly improbably random fluctuations in a prebiotic soup can result in a particularly negative impact on a student's self-esteem.

House Representative Timothy E. Macko State of New Mexico House Bill 1321 (1997)

Just as Rep. Macko is concerned for the self-esteem of children in the classrooms, we worry that the preceding analysis may have a chilling effect on the self-esteem of plasmids in bacteria. Are plasmids-like childrenmere genetic accidents, blindly formed by hapless chance and evolutionarily doomed? Fortunately, we be- lieve that there are convincing arguments to the contrary.

Selective sweeps: In bacterial populations, the appearance by mutation or immigration of a new cell carrying a selected gene may result in a selective sweep or periodic selection event in a particular bacterial ecotype. [Here we use the term "ecotype" in the sense of Cohan $(1994 a, b)$ to refer to a population or set of populations of bacteria occupying a specific ecological niche.] In such an event, the ascent of the selected gene to high frequency purges the population of genetic diversity, not only at the locus of the selected mutation, but at all or virtually all loci (At wood et al. 1951; L evin 1981). Even in the presence of recombination and local adaptation, selective sweeps can purge populations of genetic diversity at all loci sufficiently near to the selected mutation (Cohan 1994a; Majew ski and Coh an 1999).

T u r ner et al. (1998) have conjectured that such selective sweeps may provide a mechanism for the maintenance of plasmids. The idea is that as a novel mutant or recombinant sweeps through the population, there will be a chance for the plasmid to transfer from the resident lineage, which is evolutionarily doomed, to the higher fitness lineage that is ascending. In this way, the plasmid may be able to "hitchhike" to high frequency.

In the analysis of the previous section, we assumed a single constant genetic background in the host, i.e, no selective sweeps, and showed that Assumption 1 implies that plasmids cannot persist in a variety of ecological scenarios. In this section, we eliminate the assumption of an invariant genetic background in the host and consider the effects of selective sweeps on the persistence of plasmids when the $S \& L$ condition holds. For simplicity, in this section we remove the time dependence of the parameters $\psi, \alpha, \tau$, and $\gamma$. As before, there are two cases of interest. First, we consider the case in which the plasmid does not carry beneficial genes. Second, we consider the case in which the plasmid does carry beneficial genes, but must compete with a chromosomal variant that carries the same beneficial genes without paying the cost of plasmid carriage.

Plasmids bearing no beneficial genes: $\mathrm{H}$ ow does the total frequency of plasmid-bearing cells change during the course of a selective sweep? To answer this question, we must track four populations: cells with and without the novel mutation and with and without the plasmid. We thus define the densities $W(t), W^{\prime}(t), M(t)$, and $M^{\prime}(t)$, where $W$ indicates wild type, $M$ indicates mutant, and primes (') indicate plasmid-bearing cells. We define the total density of bacteria $N(t) \equiv W(t)+$ $W^{\prime}(t)+M(t)+M^{\prime}(t) \leq N^{+}$, the density of plasmidbearing cells $P(t) \equiv W^{\prime}(t)+M^{\prime}(t)$, and the density of plasmid-free cells $F(t) \equiv N(t)-P(t)=W(t)+M(t)$. We introduce the term $b$ to represent the fractional fitness benefit conferred by the novel mutation. Thus, the growth rates of the populations $W(t), W^{\prime}(t), M(t)$, 
and $M^{\prime}(t)$ and $M^{\prime}(t)$ are $\psi, \psi(1-\alpha), \psi(1+b)$, and $\psi(1-\alpha)(1+b)$, respectively. As in previous sections, we ignore the contribution of segregants to the plasmidfree class; this assumption is convenient but does not affect our conclusions. The dynamics of the system are governed by

$$
\begin{aligned}
\dot{W}(t) & =[\psi-\gamma P-u(t)] W \\
\dot{W}^{\prime}(t) & =[\psi(1-\alpha)-u(t)-\tau] W^{\prime}+\gamma P W \\
\dot{M}(t) & =[\psi(1+b)-\gamma P-u(t)] M \\
\dot{M}^{\prime}(t) & =[\psi(1-\alpha)(1+b)-u(t)-\tau] M^{\prime}+\gamma P M .
\end{aligned}
$$

To track the progress of the plasmid, it is convenient to calculate the rate of change in the ratio of plasmidbearing to plasmid-free cells, $\theta(t)=P(t) / F(t)$. This quantity is easilycalculated from Equation 9 and is given by

$\dot{\theta}=\theta\left[\gamma \mathrm{N}-\tau-\psi \alpha+\psi b\left(\frac{\mathrm{M}^{\prime}(1-\alpha)}{\mathrm{P}}-\frac{\mathrm{M}}{\mathrm{F}}\right)\right]$.

This equation has an intuitive interpretation: the change in the ratio of plasmid-bearing to plasmid-free cells follows the usual transfer-selection-segregation dynamics ( $\gamma \mathrm{N}-\psi \alpha-\tau)$, modified by a term that combines the growth rate advantage of the mutant $\psi b$ and the linkage disequilibrium between the plasmid and the beneficial mutation, scaled by the cost of plasmid carriage $(1-\alpha)$. From the $S \& L$ criterion, $\gamma \mathrm{N}-$ $\psi \alpha-\tau$ is negative at all times. The $\psi b$ term is negative whenever the linkage disequilibrium between the plasmid and the beneficial mutation, $D(t)=\left[W(t) M^{\prime}(t)-\right.$ $\left.W^{\prime}(t) M(t)\right] / N^{2}(t)$, is negative. Since we assume that the mutation comes from outside the population (T ur ner et al. 1998), it will be on a nonplasmid-bearing cell, so at time $t=0$, linkage disequilibrium is indeed negative [because $M(0)>M^{\prime}(0)=0$ ]. It follows that the $\psi b$ term is also negative. Thus, at $t=0$, the frequency of the plasmid is decreasing. We show in appendix $c$ that if the linkage disequilibrium starts out negative, it will never become positive. Therefore, the $\psi b$ term will always be negative, and the frequency of the plasmid will continuously decrease during the selective sweep. Furthermore, since in the absence of selective sweeps, $\theta$ would decline at a rate $d \theta / d t=\gamma \mathrm{N}-\psi \alpha-\tau$, the presence of sweeps increases the rate at which the plasmid disappears and therefore decreases the persistence time of the plasmid. In summary:

Result 5. If plasmids bearing no host-beneficial genes are lost in competition with plasmid-free cells under a constant genetic background, then selective sweeps of beneficial mutations introduced from outside the population will not preserve these plasmids either. Furthermore, such selective sweeps will hasten the decline of plasmid-bearing cells or, equivalently, reduce their expected persistence time.

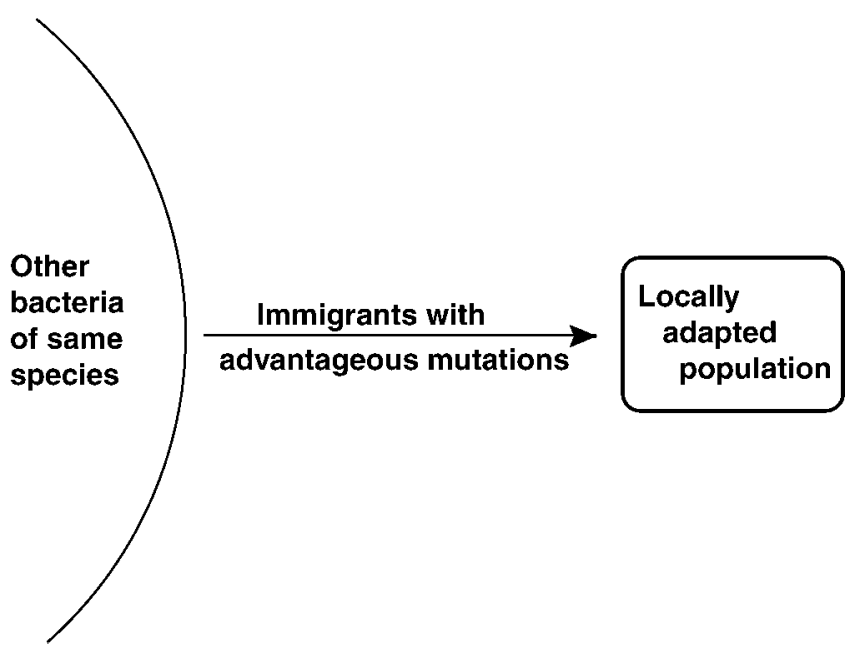

Figur e 1.-The selective sweep model: bacteria carrying highly beneficial mutations migrate into a locally adapted population.

Plasmids bearing beneficial genes: Though selective sweeps cannot facilitate the persistence of parasitic plasmids, they can maintain plasmids carrying beneficial focal genes. Consider the situation illustrated in Figure 1: a small, locally adapted population that faces a particular selective environment different from that faced by other closely related bacterial populations. By local adaptation, we mean that there is at least one gene or allele that is advantageous and has reached high frequency in the local population but that is not favored in the rest of the bacteria of the same species. The locally adaptive gene was carried on a plasmid at the time when it initially reached high frequency, and the plasmid bearing that gene reached high frequency as a result. In our earlier models, where the genetic background was constant, the appearance of a chromosomal variant ( carrying the locally adaptive gene but not the plasmid) would have resulted in the selective disappearance of the plasmid from the population. However, in the presence of selective sweeps, the plasmid might be rescued by the sequence of events shown in Figure 2 and described in detail below.

In Figure 2, only plasmid-free cells and plasmid-bearing cells, which carry a slightly beneficial, locally adaptive focal gene (represented by the lightly shaded square) are initially present in the local population. Then,

1. The plasmid-encoded focal gene (lightly shaded square) is incorporated into the chromosomal genome of some cells by transposition or some other rare genetic event, and the plasmid is lost from these cells. These newly formed "chromosomals" enjoy a selective advantage over the plasmid-bearing cells, because they gain the fitness benefits of the focal gene without the cost of the plasmid. Assuming that the $S \& L$ criterion holds, this chromosomal variant 


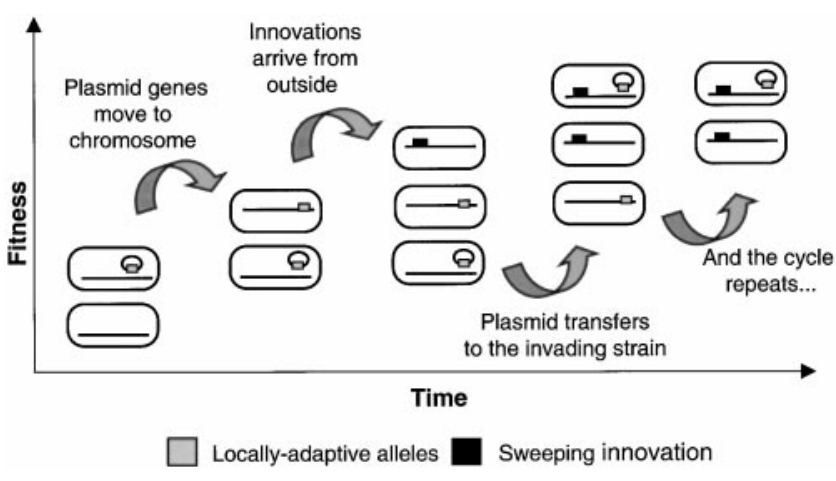

Figure 2.-The process by which plasmids can be maintained through a series of selective sweeps. See the text for a full description.

will begin to increase in frequency at the expense of the plasmid carrier.

2. Before the plasmid-bearing type is lost, however, a new strain, carrying a highly beneficial innovation (represented by a solid square) on the chromosome, but lacking the focal gene, enters the population and begins to replace the other types.

3. During this selective sweep, the new strain increases in frequency as the plasmid and chromosomal carriers of the focal gene decline. Before the plasmidbearing cells go extinct, however, the plasmid may transfer to one of the cells bearing the novel mutation, resulting in a cell that carries both the new innovation (solid square) and the focal gene (lightly shaded square).

4. The new cell with both the innovation and the focal gene will now be the most fit genotype in the population. We are in effect back where we started. If no further genetic events occur, this type will go to fixation and the frequency of the plasmid will climb (nearly) to one.

Just as before, however, a variant eventually will arise with both the new innovation and the focal gene expressed on the chromosome. Free of the cost of plasmid carriage, this type can now go to fixation, at the expense of the plasmid-bearing cells, unless another selective sweep occurs and the above process repeats.

We can observe multiple cycles of this process via computer simulation. Figure 3 shows a series of five simulated selective sweeps in a local population carrying a slightly beneficial plasmid. Figure 3 a shows the average number of novel mutations ( the mutations responsible for the selective sweeps) present in the population. Figure $3 \mathrm{~b}$ shows the frequencies of plasmid-bearing cells, chromosomals, and cells without the focal gene over time. It is assumed that the external bacterial population sequentially becomes fixed for each of five beneficial mutations, so that as time passes, immigrants from the external population to the local population have pro-

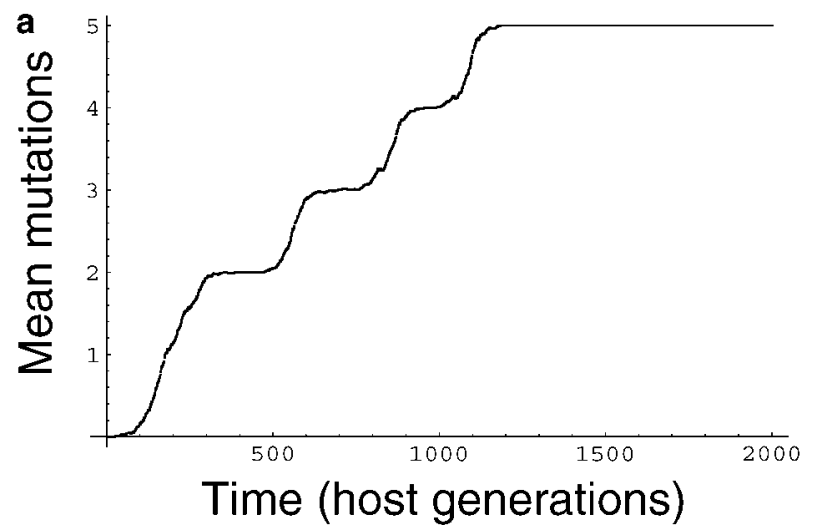

b

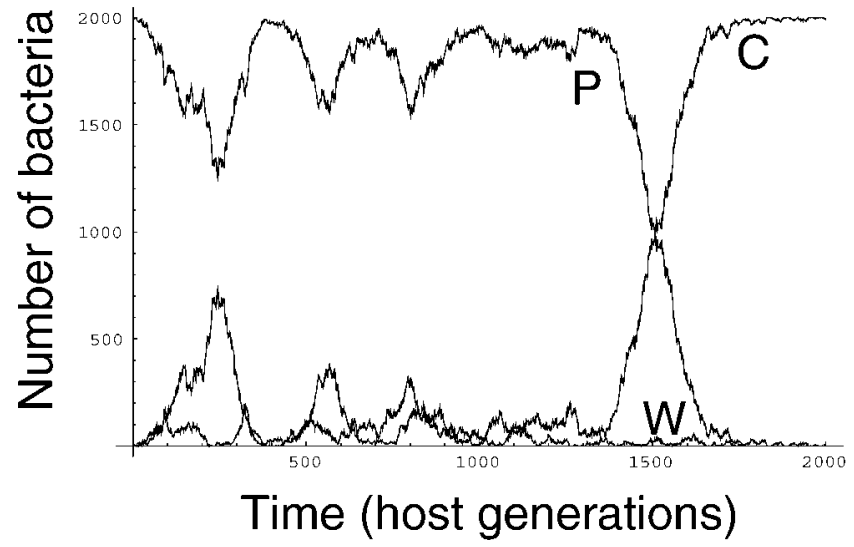

Figure 3.- Results from a computer simulation of five cycles of the process shown in Figure 2, in which selective sweeps preserve a plasmid in competition with chromosomal variants carrying the focal gene but not the plasmid. (a) The mean number of beneficial mutations in the population; each increase represents a sweep through the population of a novel beneficial mutation with selective value b. (b) The number of bacteria in the population carrying the focal gene on the plasmid (P), on the chromosome (C), or not at all (W). Parameters (chosen for illustrative and computational convenience and described in appendix d): $\mathrm{N}=2000, \psi=1, \mathrm{~V}=1, \alpha=$ $0.01, \beta=0.04, b=0.05, \gamma=0.006$ per unit volume per generation, and $x=0.0002$ per generation. An immigrant enters from outside the population on average every 2 generations. The number of beneficial mutationsfixed in the outside population starts at 1 and is incremented, on average, every 200 generations.

gressively more of these mutations, and therefore progressively higher fitness.

In the simulations, the plasmid can persist when selective sweeps are occurring; each of five novel mutations "rescues" the plasmid from extinction that it would have faced due to competition from chromosomal carriers of the focal gene. Each dip in the number of plasmidbearing cells represents the appearance and selective rise of a chromosomal variant. Subsequently, a beneficial mutation from outside appears, and the transfer of the plasmid to the mutant lineage results in an increase in plasmid numbers. To show that the sweeps are in fact necessary for plasmid persistence, we have halted 
the sweeps by stopping the appearance of novel mutations after five such mutants have appeared. As a result, the chromosomal variant that carries the focal gene and the five novel mutations can out-compete its plasmidborne counterpart, and the plasmid at last goes extinct. This simulation illustrates the need for a constant cycle of selective sweeps, with sufficient frequency to rescue the plasmid before it goes extinct.

This process can also be characterized analytically. Following the appearance of the chromosomal variant, the probability that such a "rescue" occurs before the plasmid reaches extinction is equal to the product of the probability that a mutant appears before the plasmid goes extinct, and the probability that a transfer of the plasmid to the mutant lineage then occurs during the mutant's selective sweep. (We assume that focal gene transfer from chromosomals is extremely rare and that once the plasmid transfer occurs, the transconjugant will deterministically sweep to fixation.) Let $T_{a}$ be the expected waiting time for the appearance of a chromosomal variant of a particular plasmid-borne gene, $P_{R}$ be the probability that such a rescue will occur following the appearance of a chromosomal variant, and $T_{f}$ be the expected time for the fixation of a chromosomal variant, once it has appeared and conditional that the plasmid is not rescued by a selective sweep. The expected persistence time of a plasmid in a population subject to selective sweeps is approximately

$$
T_{P}=T_{a} /\left(1-P_{R}\right)+T_{f} .
$$

Note that in the absence of selective sweeps, $P_{R}=0$ and the persistence time is simply $T_{a}+T_{f}$, the time until a chromosomal variant appears plus the time required for the fixation of that chromosomal variant.

In summary, we have:

Result 6. When a plasmid carries beneficial genes but would be out-competed within a single population by chromosomal variants carrying the same beneficial genes, the occurrence of selective sweeps following the arrival of more-fit mutants or recombinants can extend the persistence time of an infectiously transmitted plasmid.

Moreover, if the probability of a plasmid transferring across into the sweeping population exceeds that plasmid's frequency in the presweep population, then the expected frequency of the plasmid actually increases over time. When plasmids carry no genes useful to the host, by contrast, the expected frequency of the plasmid never increases, as proved above.

Figure 4 shows how $T_{p}$ depends on the rate of appearance of new mutants of the relevant type and other parameters. The methods for calculating $T_{p}$ are given in appendix $d$. Finally, it is interesting to note the similarity between this scenario, which favors plasmids, and the scenario suggested by Maynard Smith et al. (1993) as a possible advantage to chromosomal recombination:
"In pathogenic bacteria, at least, the capacity for localized recombination may be essential for the maintenance of useful variation (e.g., in cell surface genes) within the population against the purifying effects of repeated waves of periodic selection."

Cross-ecotype transfer: In the previous section, we showed how plasmids can persist by transferring genes across selective sweeps. In this section, we explore how plasmids can persist by transferring genes beyond the range of selective sweeps (and other purifying events). We do not attempt a general treatment, but instead merely illustrate that plasmids can persist in a multipleecotype model even when Assumption 1 is met. The reader may note that this model is closely related to standard models in metapopulation theory ( $\mathrm{H}$ anski and G il pin 1997; H anski 1999), with the plasmids playing the role of the "organism" and distinct bacterial ecotypes or species (rather than spatial locations) playing the roles of the "habitats" that jointly comprise the metapopulation.

In the interest of simplicity, the example presented in this section is a discrete-time model featuring only two host ecotypes. The underlying dynamics, however, will generalize to continuous time, large numbers of ecotypes, and more complex temporal and spatial fitness fluctuations. Of course, models with more ecotypes and wider ranges of conditions may more realistically portray the natural ecology of bacterial plasmids.

Consider a focal gene that codes for antibiotic resistance (for example) in two distinct bacterial ecotypes, labeled A and B. In each ecotype, "resistant" individuals carrying the focal gene typically have a fitness of $1+\beta$ relative to "sensitive" individuals that lack this gene, where $\beta>0$ represents the fitness advantage due to resistance. The focal gene may be chromosomal or plasmid-encoded; when it is the latter, the cost of plasmid carriage imposes a fitness reduction of $(1-\alpha)$ on the host bacterium, for a total fitness of $(1+\beta)(1-\alpha)$. O ccasionally, due to various purifying mechanisms (demographic stochasticity, population bottlenecks, selective sweeps at other loci, environmental fluctuations leading to strong selection against the focal gene, etc.), the focal gene will be lost entirely from one ecotype; typically the other will be unaffected (M aynar d Smit h 1991; Cohan 1994a; Pal ys et al. 1997). Let this occur with probability $k$ in each ecotype and assume, for simplicity, that loss never occurs simultaneously in both ecotypes. Finally, assume that a fraction $z$ of individuals carrying the plasmid engage in cross-ecotype plasmid transfer to sensitive hosts; this process is summarized by the matrix $\mathcal{T}$. Here we are treating crossecotype transfer as a deterministic process and considering $\mathscr{T}$ to be the matrix of transfer frequencies; we could alternatively consider a stochastic process of cross-ecotype transfer, in which $\mathscr{T}$ would represent transfer probabilities.

Let $c_{A}(t)$ denote the ratio of cells with chromosomal 


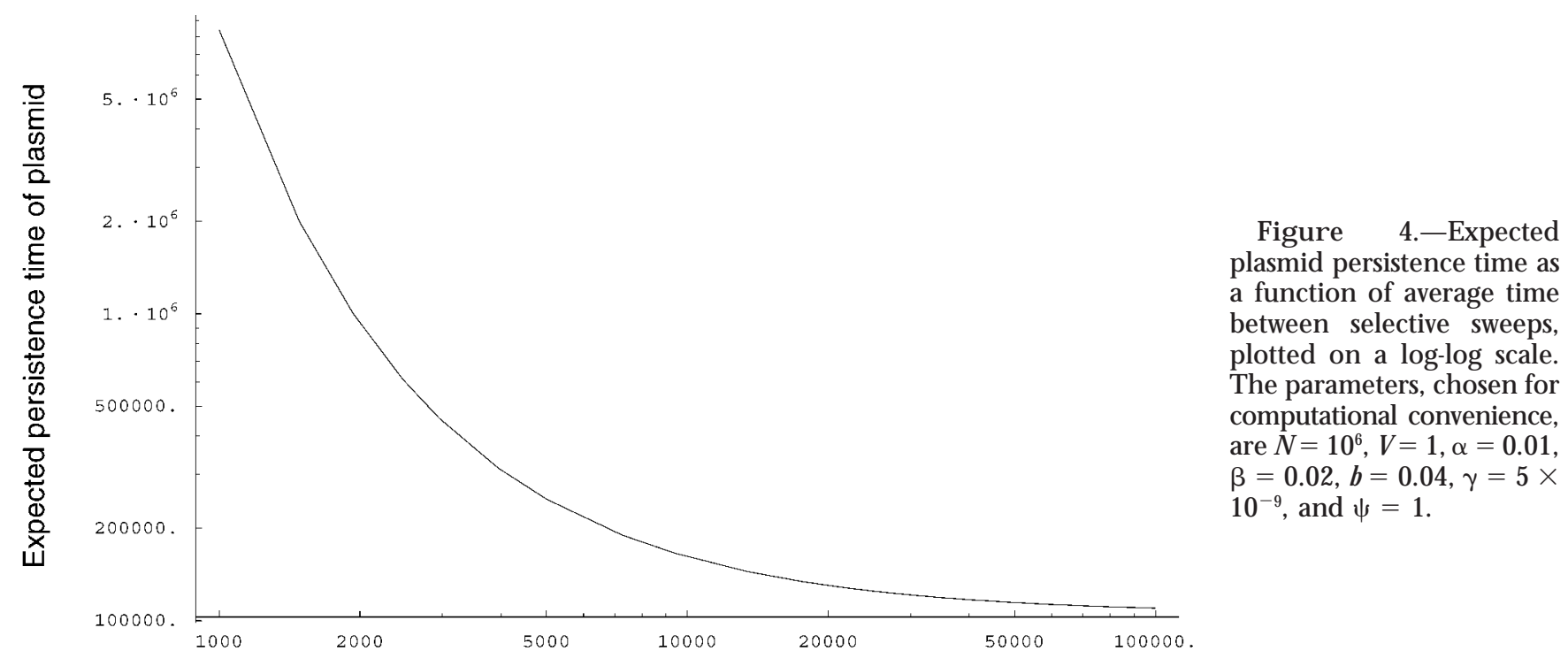

Average time between selective sweeps

resistance to cells that are sensitive at time $t$ for ecotype $A$, let $c_{B}(t)$ represent the equivalent ratio in ecotype $B$, and define the column vector $c(t) \equiv\left(c_{A}(t), c_{B}(t)\right)$. Similarly, let $p(t) \equiv\left(p_{A}(t), p_{B}(t)\right)$ be the ratios of plasmidbearing resistant cells to sensitive cells in ecotypes $A$ and $B$. Assume that transfer for the focal genes from plasmid to chromosome by transposition or alternative processes is very slow relative to the rate of loss of the focal gene from a specific habitat. The ratios at time $t+1$ are then

$$
\begin{aligned}
& c(t+1)=\eta c(t) \\
& p(t+1)=(1-\alpha) \eta \mathscr{T} p(t),
\end{aligned}
$$

where

and

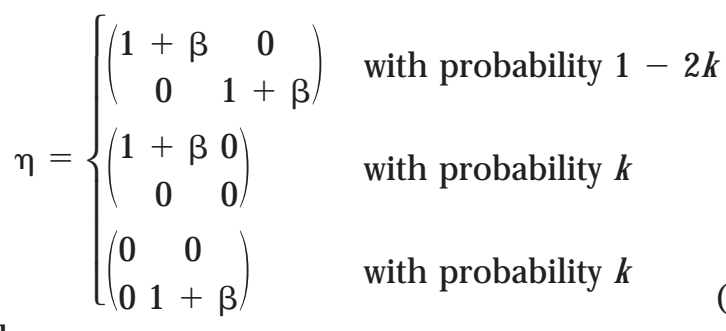

$$
\mathscr{T}=\left(\begin{array}{cc}
1-z & z \\
z & 1-z
\end{array}\right)
$$

Result 7 then follows directly.

Result 7. In this illustrative model, chromosomals are al ways locally favored over plasmids. H owever, chromosomals cannot persist in the long run whereas plasmids will be able to persist due to their potential for cross-species transfer.

Figure 5 illustrates the dynamics of this system. Whenever they are present, chromosomals outperform plas-
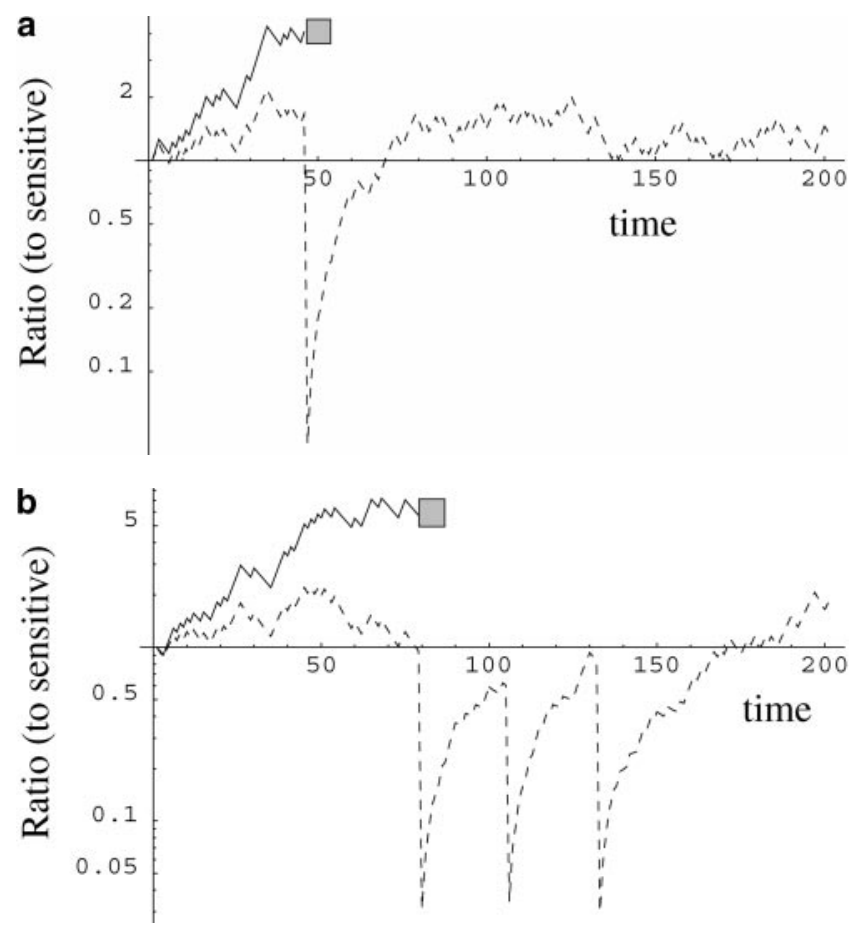

Figure 5.-A computer simulation of the two-ecotype model. To improve clarity, we have replaced the fitness parameter $\beta$ with a distribution of fitnesses. Solid lines represent the ratio of chromosomals to sensitives, shown on a log scale, in (a) ecotype A and (b) ecotype B. Dotted lines represent ratio of plasmid-bearing cells to sensitives, on a log scale, in each ecotype. Environmental conditions are selected randomly each time period. Starting ratios are 1:1 for both chromosomals and plasmid-bearing cells in both ecotypes. Parameter values ( see appendix $d$ ) are $k=0.01, r=0.02$, and $t=$ 0.02 . The value of $\beta$ fluctuates: $\beta=0.125$ or $\beta=-0.05$, each with probability $1 / 2$. Extinction events (and loss of the chromosomals in one ecotype) are marked with a shaded square. 
mid-bearing cells; nevertheless, plasmids persist whereas chromosomals are lost. This happens because the extinction events clear all copies of the focal gene from a particular ecotype. The plasmid-encoded form of the gene is able to subsequently "recolonize" following clearance, whereas the chromosomals cannot be regenerated once lost from a given ecotype. Asa consequence, after some time, the focal genes are carried exclusively on the plasmid.

\section{DISCUSSION}

In this article, we have examined the existence conditionsfor bacterial plasmids, the conditions under which plasmids can become established and will be maintained in populations and communities of bacteria. Throughout our analysis, we have operated on the assumption that in populations of realistic density, these elements transfer at too low a rate to overcome the joint effects of segregation and selection. As such, plasmids' establishment and persistence depends their being "nice" to their hosts by carrying genes that at least occasionally augment the fitness of the bacteria carrying them, e.g., genes for antibiotic resistance, virulence, or fermentation of unusual carbon sources. We also have assumed that by transposition or other recombinatory mechanisms, these beneficial genescan move from the plasmid to the host chromosome, where they engender less cost when they are not needed and greater benefits when they are.

Using a general model, we have shown that when these assumptions are met, plasmids cannot be maintained indefinitely in single-clone populations of bacteria even when selection favors the genes that they carry. While bacteria carrying plasmids can ascend and do well for a while, the genes responsible for their good fortune will eventually make their wayto the chromosome. Eventually, the plasmid will be lost. Indeed, these symbiotic plasmids find themselves in a bind, trapped between carrying genes that are too useful (in which case those genes will be rapidly incorporated into the chromosome) and carrying genes that are too useless (in which case the plasmid-bearing cells will be out-competed by plasmid-free ones.) In accord with our analysis, this sad situation is anticipated (1) in a single population of bacteria under constant or fluctuating selection and (2) in a metapopulation with intermittent selection favoring plasmid-borne genes in different subpopulations at different times.

N evertheless, our results are not entirely inconsistent with plasmids' existence. Employing more specific models, we have found conditions under which infectiously transmitted plasmids can be maintained indefinitely in the face of chromosomal imperialism. O ne process that can facilitate plasmid persistence is the action of selective sweeps. Even in initially homogeneous populations residing in unchanging habitats, higher fitness variants will be generated periodically by mutation or by the receipt of favored genes and accessory elements from without, and these new variants will sweep through the population (Atwood et al. 1951; Lenski et al. 1991; Travisano et al. 1995; El ena et al. 1996). Because of their capacity for infectious transfer, plasmids are more likely than chromosomal genes to become associated with incoming favorable mutants and ascend to prominence by hitchhiking. Our results indicate, however, that to hitchhike successfully the plasmid must share the driving; it must carry genes that are beneficial with sufficient frequency to outweigh the cost of their carriage. Moreover, there has to be a fair amount of traffic on the road for the plasmid to hitchhike for a long distance. If periodic sweeps are rare, then the "focal" gene giving plasmid its advantage will make its way to the chromosome and the chromosomal variants will force the plasmid into extinction before it is rescued by a selective sweep. If sweeps are common, on the other hand, a new sweep will rescue the plasmid from this fate, and thus the plasmid can be maintained for a considerable period.

Alternatively (or in addition), plasmids may persist because of their ability to shuttle genes across ecotype or species boundaries. In this scenario, functional genes borne on plasmids disperse horizontally in genotype space, "outrunning" the boundaries of any particular selective sweep. An important virtue of crossecotype transfer in taxa for which ecotypes are perhaps best defined by the range of selective sweeps (Maynard Smit h 1991; C o h an 1994a,b, 1996) is the ability to avoid eradication by any particular sweeping clone or genotype.

Of course, several caveats are necessary when interpreting the results of this article. Perhaps the most important of these is that all of the results derived in part $i$ are based on the assumption that plasmids cannot be maintained as genetic parasites in bacterial populations of realistic densities and turnover rates, because their rate of infectious transmission is insufficient to overcome selection and segregation. While the evidence generated from estimates of the rates of plasmid transfer, fitness costs, and rates of vegetative segregation generally supports this assumption, it is possible that the limited number of laboratory studies that actually estimated these parameters do not reflect what real plasmids do in the real world. It may well be that some plasmids can persist as purely parasitic genetic elements in natural populations. The major limitations of existing investigations of estimating these parameters are that they typically (1) are restricted to liquid culture with bacteria persisting as individual cells, whereas in much of the real world bacteria exist as microcolonies on surfaces, semisolids, and biofilms, for which the dynamics of transfer are different as may be the costs of their carriage and rates of segregation (but see Simonsen 1990); (2) ignore transitory derepression of conjugative 
pili synthesis, where cells that recently acquired plasmids transfer them at higher rates than cells from lineages that have carried them for some time [though Lundquist and Levin (1986) is an exception]; and (3) use laboratory strains rather than wild plasmids and/ or wild bacterial hosts. It is clear that conjugation rates vary widely among plasmids and their bacterial hosts (Gordon 1992) and there may well be plasmid-host pairsfor which the rate parameters of infectious transfer are far greater than those al ready published. M oreover, natural selection on both the host and plasmid would, if all else were equal, favor mechanisms that reduce the fitness burden of plasmid carriage, and selection on the plasmid would favor mechanisms that minimize these rates of loss by vegetative segregation, such as high copy number and postsegregational killing. Consequently, although a plasmid in a particular host may initially impose a substantial burden and suffer loss at a high rate, these costs and segregation rates are likely to evolve to lower and possibly even negligible levels.

Even if plasmids can be maintained as "genetic parasites," however, this explanation for their long-term persistence will still not account for the observation that certain types of functional genes (e.g., those for antibiotic resistance) are commonly borne on plasmids. Accounting for these patterns of genome organization will require some sort of ecological theory similar to that considered here.

A second caveat involves the existence of the so-called cryptic plasmids. Many plasmids, including the majority borne by some species such as Escherichia coli ( Caugant et al. 1981), are "cryptic" (Novick et al. 1976) in the sense that they do not appear to carry any genes other than those necessary for their own maintenance and transmission. At first glance, this seems inconsistent with the mechanisms of plasmid maintenance considered here, which require the carriage of genes that at least occasionallyaugment the fitness of their host bacterium. We see three possible ways to account for the existence of these cryptic plasmids. First, these plasmids might not really be cryptic; perhaps we just have not yet discovered the ways in which they augment the fitness of the bacteria that carry them. Second, cryptic plasmids may have evolved to be sufficiently innocuous and stable to be maintained by even low rates of infectious transfer, i.e., to persist as genetic parasites. Third, the prevalence of cryptic plasmids could be a nonequilibrium phenomenon; these plasmids once carried useful genes, recently lost them, and will either become extinct or acquire useful genes in sufficient time to continue to persist by the mechanisms considered here. In thisinterpretation, there would be a balance between the rates of acquisition and loss of host-beneficial genes by plasmids.

It is important to realize that the models presented in this article are fundamentally ecological, rather than genetic: allele frequencies may change but the genes under consideration do not. Our models are con- strained so as to forbid introduction of novel variation at the focal (plasmid-encoded) loci, and, in part i, at nonfocal loci as well. In practice, the specifics of the plasmid-encoded lifestyle (e.g., copy number and horizontal transfer) could have dramatic evolutionary consequences for the generation and maintenance of variation at plasmid loci. More sophisticated models will be required to evaluate the possibility that these factors do play an important role in explaining plasmid persistence.

The analysis presented here provides a set of preliminary hints or suggestions for possible answers to the question, "What sorts of genes should be on plasmids?" As we have shown, basic ecological characteristics of the focal genes (the variation across ecotypes in their selective consequences at a given time t, the ease by which they can be generated by mutation, the probability of their loss from given species, the correlation in the probability of loss at a given time across species, etc.) determine for these genes the magnitude of the advantage or disadvantage of being carried on a plasmid. For example, when selective sweeps by immigrating strains are common, we might expect that plasmids will carry locally adapted genes and that plasmids may in fact be important in facilitating local adaptation in asexual haploids. When cross-ecotype transfer plays an important part in plasmid persistence, we might expect plasmids to carry sometimes beneficial genes that cannotfor whatever reason-be maintained in single-clone populations. However, these are merely suggestions of the present model; more detailed analysis will be necessary to answer the question properly. We therefore believe that further exploration of such questions will be a productive and perhaps even essential avenue in our efforts to understand the population and evolutionary biology of plasmids.

The authors thank a number of individuals for their helpful discussion, comments, and mathematical assistance: R. Antia, J. Paulsson, A. Robson, J. Smith, and M. Tanaka. A. Clark and two anonymous referees provided a number of valuable suggestions. $\mathrm{C}$. Bergstrom is partially supported by National Institutes of $\mathrm{H}$ ealth $(\mathrm{NIH}) / \mathrm{N}$ ational Institutes of Allergy and Infectious Diseases grant T32-AI0742. M. Lipsitch was supported by NIH grant F32-GM19182. B. Levin was supported by NIH grant F32-GM 33782.

\section{LITERATURE CITED}

Atwood, K. C., L. K. Schneider and F. J. Ryan, 1951 Periodic selection in Escherichia coli. Proc. Natl. Acad. Sci. USA 37: 146-155.

Campbel I, A. M., 1961 Conditions for the existence of bacteriophage. Evolution 15: 153-165.

Caugant, D. A., B. R. Levin and R. K. Sel ander, 1981 Genetic diversity and temporal variation in $\mathrm{E}$. coli population of a human host. Genetics 98: 467-490.

Cohan, F. M., 1994a The effect of rare but promiscuous gene exchange on evolutionary divergences in prokaryotes. Am. Nat. 143: 965-986.

Cohan, F.M., 1994b Genetic exchange and evolutionary divergence in prokaryotes. Trends Ecol. Evol. 9: 175-180.

Cohan, F.M., 1996 The role of genetic exchange in bacterial evolution. ASM News 62: 631-636. 
Eberhard, W. G., 1990 Evolution in bacterial plasmids and levels of selection. Q. Rev. Biol. 65: 3-22.

El ena, S. F., V.S. Cooper and R. E. Lenski, 1996 Punctuated evolution caused by selection of rare beneficial mutations. Science 272: 1802-1804.

Gerdes, K., P. B. Rasmussen and S. Mol in, 1986 Unique type of plasmid maintenance function: postsegregational killing of plasmid-free cells. Proc. Natl. Acad. Sci. USA 83: 3116-3120.

Gill espie, J., 1973 Polymorphism in random environments. Theor. Popul. Biol. 4: 193-195.

Gordon, D. M., 1992 Rate of plasmid transfer among Eschericia coli strains isolated from natural populations. J. Gen. Microbiol. 138: $17-21$.

H al dane, J. B. S., and S. D. Jayakar, 1963 Polymorphism due to selection of varying direction. J. Genet. 58: 237-242.

H anski, I., 1999 Metapopulation dynamics. Nature 396: 41-49.

H anski, I., and M. E. Gil pin, 1997 Metapopulation Biology. Academic Press, New York.

Hofbauer, J., and K. Sigmund, 1988 The Theory of Evolution and Dynamical Systems: M athematical A spects of Selection. Cambridge U niversity Press, Cambridge, United Kingdom.

I ppen-Ih I er , K., 1989 Bacterial conjugation, pp. 32-72 in GeneT ransfer in the Environment, edited by S. B. Levy and R. B. M iller. McGraw-Hill, New York.

Lenski, R. E., M. R. Rose, S. C. Simpson and S. C. Tadler, 1991 Long-term experimental evolution in Escherichia coli. I. Adaptation and divergence during 2000 generations. Am. Nat. 91: 1315-1341.

Levin, B. R., 1980 Conditions for the existence of R-plasmids in bacterial population, pp. 197-202 in Proceedings of the Fourth International Symposium on Antibiotic R esistance. Springer-Verlag, Berlin.

Levin, B. R., 1981 Periodic selection, infectious gene exchange, and the genetic structure of E. coli populations. Genetics 99: 1-23.

Levin, B. R., 1993 The maintenance of genetic variation in bacterial populations, pp. 193-200 in Genetics of Cellular, Individual, Family, and Population Variation, edited by C. F. Sing and C. L. H anis. Oxford U niversity Press, Oxford.

Levin, B. R., and C. T. Bergst rom, 2000 Bacterial are different: observations, interpretations, speculations, and opinions about the mechanisms of adaptive evolution in prokaryotes. Proc. Natl. Acad. Sci. USA 97: 6981-6985.

Levin, B. R., and V. A. Rice, 1980 The kinetics of conjugative plasmids by mobilizing conjugative factors. Genet. Res. 35: 241-259.

Levin, B. R., F. M. St ewart and V. A. Rice, 1979 The kinetics of conjugative plasmid transmission: fit of a simple mass-action model. Plasmid 2: 247-260.

Lundquist, P. D., and B. R. Levin, 1986 Transitory derepression and the maintenance of conjugative plasmids. Genetics 113: 483497.

Luria, S. E., and J. L. Suit, 1987 Colicins and Col plasmids, pp. 1615-1624 in Escherichia coli and Salmonella typhimurium: Cellular and M olecular Biology, edited by J. Ingr a ham, K. B. Low, B. M agasanik, M. Schaechter, H. E. U mbarger and F. C. Neidhardt. ASM Press, Washington, DC.

Majewski, J., and F. M. Cohan, 1999 Adapt globally, act locally: the effect of selective sweeps on bacterial sequence divergence. Genetics 152: 1459-1474.

Maynard Smit h, J., 1991 The population genetics of bacterial. Proc. R. Soc. Lond. Ser. B 245: 37-41.

Maynard Smith, J., N. H. Smith, M. O'Rourke and B. G. Sprat t, 1993 How clonal are bacteria? Proc. Natl. Acad. Sci. USA 90: 4384-4388.

Mongold, J.A., 1992 Theoretical implications for the evolution of postsegregational killing by bacterial plasmids. Am. Nat. 139: 677-689.

Nor dst röm, K., and S. J. A ust in, 1989 Mechanisms that contribute to the stable segregation of plasmids. Annu. Rev. Genet. 23: 37-69.

Nordst röm, K. Mol in and J. Light, 1984 Control of replication of bacterial plasmids: genetics, molecular biology, and physiology of the plasmid R1 system. Plasmid 12: 71-90.

Novick, R. P., R. C. Cl owes, S. N. Cohen, R. Curt iss, N. Dat t a et al., 1976 Uniform nomenclature for bacterial plasmids: a proposal. Bacteriol. Rev. 40: 168-189.

Pal ys, T., L. K. Nakamura and F. M. Cohan, 1997 Discovery and classification of ecological diversity in the bacterial world: the role of DNA sequence data. Int. J. Syst. Bacteriol. 47: 1145-1156.
Paulsson, J., and M. Ehr enber g, 1998 Trade-off between segregational stability and metabolic burden: a mathematical model of plasmid ColE1 replication control. J. Mol. Biol. 279: 73-88.

Simonsen, L., 1990 Dynamics of plasmid transfer on surfaces. J. Gen. Microbiol. 136: 1001-1007.

Simonsen, L., 1991 The existence conditions for bacterial plasmids: theory and reality. Microb. Ecol. 22: 187-205.

Simonsen, L., D. M. Gordon, F. M. St ewart and B. R. Levin, 1990 Estimating the rate of plasmid transfer: an end-point method. J. Gen. Microbiol. 136: 2319-2325.

St ewart, F. M., and B. R. Levin, 1977 The population biology of bacterial plasmids: a priori conditions for the existence of conjugationally transmitted factors. Genetics 87: 209-228.

Summer S, D. K., 1996 The Biology of Plasmids, Blackwell Science Ltd., Oxford.

Travisano, M., J. A. Mongold, A. F. Bennett and R. E. Lenski, 1995 Experimental tests of the roles of adaptation, chance, and history in evolution. Science 267: 87-90.

Turner, P. E., V. S. Cooper and R. E. Lenski, 1998 Tradeoff between horizontal and vertical modes of transmission in bacterial plasmids. Evolution 52: 315-329.

Yosh imura, J., and V. A. A. Jansen, 1996 Evolution and population dynamics in stochastic environments. Res. Popul. Ecol. 38: 165182.

Communicating editor: A. G. Cl ark

\section{APPENDIX A}

What happens if we relax the assumptions regarding the cost $\alpha$ of carrying the plasmid? In particular, consider the case in which $\alpha$ is also a function of t, subject to an extended version of the $S \& L$ criterion:

$$
\psi(\mathrm{t}) \alpha(\mathrm{t})+\tau(\mathrm{t})>\gamma(\mathrm{t}) \mathrm{N}^{+} .
$$

This opensup a new possibility for the plasmids: Though still subject to the $S \& L$ criterion, they are now allowed to facultatively adjust their carriage costs ( perhaps by shutting down the conjugation-related genes under some conditions and by turning them on under others) in response to the values of $\psi(t)$ and $\beta(t)$. In this way, a plasmid could theoretically code for high transfer at high cost under some circumstances and low transfer at low cost under other circumstances.

Can this allow plasmid persistence? When the selective consequence of the "focal genes," $\beta(t)$, fluctuates widely from positive to negative, it can. The Stewart and Levin criterion merely states that vertical transmission is more effective than is horizontal transmission in the absence of any fitness effects of the focal genes. When the focal genes' effects are sufficiently deleterious, horizontal transmission could become more effective than vertical transmission without violating the Stewart and Levin criterion. Under these circumstances, a plasmid could persist via facultative conjugation, reaping an advantage by carrying often-but-not-always beneficial genes and by facultatively switching to horizontal transmission when opportunities for vertical transmission are limited by temporarily adverse consequences of the focal genes.

In terms of the model, this would require that high transfer rates $\gamma(\mathrm{t})$ at high $\cos t \alpha(\mathrm{t})$ be tightly correlated with conditions of low growth $[\beta(t)$ near -1$]$. Under 
these circumstances, plasmid-bearing cells can indeed enjoy a higher long-term growth rate than either plasmid-free or chromosomal cells. This obtains even though at any specific time, the plasmid-bearing strain will be growing at a slower rate than at least one of its competitors.

H owever, we consider this to be a formal possibility of the model, rather than a likely explanation of plasmid persistence. First, this mechanism requires enormous fluctuation in the selective effect $\beta(t)$ to be viable. Second, and more importantly, this mechanism works only because whatever biochemical processes are acting to temporarily reduce host replication rate during periods when $\beta(t)$ is near -1 are assumed not to constrain the conjugation rate. This is in contrast to the usual empirical observations that the conjugation rate is severely limited when the bacterial hosts do not replicate (Levin et al. 1979). Nevertheless, if mechanisms for extensive stationary-phase conjugation are discovered, this possibility could merit further consideration.

\section{APPENDIX B}

We sketch a proof of Result 4: If plasmids cannot be maintained as genetic parasites, then they cannot be maintained even when they carry sometimes-useful genes in a model incorporating migration among multiple habitats, with fluctuating selective conditionsin each habitat. We emphasize the caveat that this result is based on a deterministic metapopulation model with continuous-time population growth. It would be interesting to determine whether similar results will hold for stochastic migration models with population growth treated as a branching process.

We assume that conjugation rate and segregation rate are proportional to growth rate; i.e., $\gamma(\mathrm{t})$ and $\tau(\mathrm{t})$ become $\gamma(1+\beta(t))$ and $\tau(1+\beta(t))$, respectively, as growth rate changes due to fluctuations in $\beta$. Multiplying the $S \& L$ condition by the positive scalar ( $1+$ $\beta)$, we get the following expression, which holds at all timest:

$\psi(t) \alpha(1+\beta(t))+\tau(t)(1+\beta(t))>\gamma(t)(1+\beta(t)) N^{+}$.

Consider a metapopulation model of $\mathrm{h}$ local habitats, each of which is subject to fluctuating selection. Migration events (movement of one or more bacteria to another habitat) occur at discrete time points $t_{1}, t_{2}, t_{3}, \ldots$, etc. Each migration event can be described by a matrix $\phi\left(t_{n}\right)$, where $\left[\phi\left(t_{n}\right)\right]_{i j}$ is a fraction of inhabitants of habitat j migrating to habitat $i$ at time $t_{n}$. Expression (B1) ensures that in between migration events, in each habitat $i$, $\dot{\mathrm{C}}_{\mathrm{i}}(\mathrm{t}) / \mathrm{C}_{\mathrm{i}}(\mathrm{T})>\dot{\mathrm{P}}_{\mathrm{i}}(\mathrm{t}) / \mathrm{P}_{\mathrm{i}}(\mathrm{t})$; i.e, chromosomal populations grow at a faster rate (or decline at a slower rate) in each and every habitat. Migration can move individuals among habitats, but cannot, in the long run, reverse so great a disadvantage to the plasmid-bearing cells. Sub- ject to mild nondegeneracy conditions, it follows in relatively straightforward fashion that the fraction of plasmid-bearing individuals will decline and ultimately vanish over time in the metapopulation.

\section{APPENDIX C}

We prove that when a plasmid is parasitic and a selective sweep is in progress, if the linkage disequilibrium between the plasmid and the new beneficial mutation is negative, it will remain negative.

The system is described by Equation 9 in the text. The denominator of the linkage disequilibrium $D(t)=$ $\left(W(t) M^{\prime}(t)-W^{\prime}(t) M(t)\right) / N^{2}(t)$ is al ways positive, so $D(t)$ has the sign of its numerator, which we call $\Delta(\mathrm{t})=\mathrm{W}(\mathrm{t}) \mathrm{M}^{\prime}(\mathrm{t})-\mathrm{W}^{\prime}(\mathrm{t}) \mathrm{M}(\mathrm{t})$. The time derivative of $\Delta$ can be obtained easily from Equation 9 , and it simplifies to

$$
\dot{\Delta}=[\psi(2+b-\alpha)-\tau-\gamma \mathrm{P}] \Delta-\psi \mathrm{M}{ }^{\prime} \mathrm{Wb} \alpha .
$$

In the neighborhood of $\Delta=0, \dot{\Delta}$ is negative, because all terms except the last are 0 , and the last is negative. Thus, $\Delta$ is decreasing in the neighborhood of 0 . Because all quantities in this model are continuous, if $\Delta(0)<$ 0 then $\Delta(\mathrm{t})<0$ for all $\mathrm{t}>0$.

\section{APPENDIX D}

We calculate $T_{p}$, the expected persistence time of a plasmid in a population with selective sweeps. We do so by calculating in turn the elements of Equation 11 in the text, which defines $T_{p}$. Most of the effort (up through Equation D13) is in calculating $P_{R}$; we then give expressions for $T_{a}$ and $T_{f}$.

First, we calculate $P_{R}$, the probability that, following the appearance of a chromosomal variant, the plasmid is rescued by a selective sweep before it goes extinct. Initially, let us assume that the chromosomal variant arises at time $\mathrm{t}=0$.

Then, note that $P_{R}$ is given by

$$
P_{R}=\int_{0}^{\infty} P_{M}(t) P_{x}(t) d t,
$$

where $P_{M}(t)$ is the probability that the first mutant appears during the interval $[t, t+d t)$ following the appearance of the chromosomal, and $\mathrm{P}_{\mathrm{x}}(\mathrm{t})$ is the probability that a plasmid is transferred into the mutant lineage during its selective sweep, given that the sweep began in the interval $[t, t+d t)$.

Lacking a good reason to make a more complex assumption, we assume that new mutations arrive in the population as a Poisson process with a constant rate $\sigma$. Then

$$
P_{M}(t)=\sigma e^{-\sigma t}
$$

To calculate $P_{x}(t)$ we use the following model. Consider a locally adapted population of fixed density $\mathrm{N}$ in a volume V, so that there are NV bacteria present. The 
population consists initially of bacteria bearing the focal gene on a plasmid, which have density $\mathrm{P}(\mathrm{t})$ at time $\mathrm{t}$, and bacteria bearing the focal gene on their chromosome, which have density $C(t)$. We assume that all fitness effects are multiplicative, that the fitness advantage of the focal gene is $\beta$, and that the fitness cost of the plasmid is $\alpha$. Then the chromosomal and plasmid-bearing populations have growth rates $\psi(1+\beta)$ and $\psi(1+\beta)(1-$ $\alpha)$, respectively. Following the appearance of a mutant, we track the density of mutants in the population as $M(t)$, and we assume that the mutation it bears carries fitness advantage $b$, giving it a growth rate of $\psi(1+b)$, since it bears neither the focal gene nor the plasmid. For mathematical simplicity, we neglect segregation, which does not change the qualitative results. The values of these parameters are constrained by the following assumptions:

1. The fitness benefit of the focal gene is greater than the fitness cost of the plasmid: $\beta>\alpha /(1-\alpha)$.

2. The fitness benefit of the new mutation is greater than that of the focal gene (so that the focal gene will go to fixation): $b>\beta$.

3. The consequence of the Stewart and Levin criterion: the plasmid could not persist in competition with the chromosomal version by making up for its fitness burden by transfer: $\gamma \mathrm{N}<\psi \alpha(1+\beta)$.

To model changes in the densities ( tracked by capital letters) of plasmid-bearers, chromosomals, and mutants (ignoring transconjugants for the moment), imposing a constraint of constant population size, we have

$$
\begin{aligned}
& \frac{d P(t)}{d t}=[\psi(1-\alpha)(1+\beta)-\bar{\psi}] P+\gamma P C \\
& \frac{d C(t)}{d t}=[\psi(1+\beta)-\bar{\psi}] C-\gamma P C \\
& \frac{d M(t)}{d t}=[\psi(1+b)-\bar{\psi}] M,
\end{aligned}
$$

where $\bar{\psi}(t)=\psi[(1-\alpha)(1+\beta) P(t)+(1+\beta) C(t)+$ $(1+b) M(t)] / N$ is the weighted average growth rate in the population at time $t$ and is subtracted from the individual growth rates to maintain constant population size.

We assume that the chromosomal appears at $t=0$ in a single bacterium; this corresponds to an initial density of $\mathrm{C}(0)=1 / \mathrm{V}$. Following the appearance of the chromosomal, but before a mutant enters the population, we consider only the frequencies of chromosomal and plasmid-bearing types. During this period, we track the frequency of the plasmid-bearing type, $p=P /(P+$ $C)$, which can be calculated from (D3) and (D4) as

$$
\frac{d p(t)}{d t}=[\gamma N-\Psi \alpha(1+\beta)] p(1-p) .
$$

This has the solution

$$
p(t)=\frac{(N V-1) e^{[\gamma N-\Psi \alpha(1+\beta)] t}}{1+(N V-1) e^{[\gamma N-\Psi \alpha(1+\beta)] t}} .
$$

Assume the mutant appears at a particular time $t=$ $t^{*}$. At the time of the appearance of the mutant, the densities of plasmid-bearers, chromosomals, and mutants, respectively, in the population are given by

$$
\begin{aligned}
\mathrm{P}\left(\mathrm{t}^{*}\right) & =(\mathrm{N}-1 / \mathrm{V}) \mathrm{p}\left(\mathrm{t}^{*}\right) \\
\mathrm{C}\left(\mathrm{t}^{*}\right) & =(\mathrm{N}-1 / \mathrm{V})\left(1-\mathrm{p}\left(\mathrm{t}^{*}\right)\right) \\
\mathrm{M}\left(\mathrm{t}^{*}\right) & =1 / \mathrm{V},
\end{aligned}
$$

where $p\left(t^{*}\right)$ is given by (D7). Following the appearance of the mutant, the dynamics of these three populations are given by Equations D3-D5; these dynamics consist of an increase in the number of mutants (the selective sweep) while the number of plasmid-bearers and chromosomals declines.

We want to calculate $P_{x}$, the probability that the plasmid is transferred at least once to the mutant population, creating a transconjugant, before the plasmid-bearers go extinct. We use the expected number of transconjugants created during the sweep to calculate the probability of producing at least one transconjugant during the sweep. The instantaneous rate of appearance of transconjugants during this sweep is $\gamma \mathrm{P}(\mathrm{t}) \mathrm{M}(\mathrm{t})$, so $X(t *)$, the expected number of transconjugants in a selective sweep beginning at time $t=t *$, is given by

$$
X\left(t^{*}\right)=\gamma \int_{\mathrm{t}^{*}}^{\infty} \mathrm{P}(\mathrm{s}) \mathrm{M}(\mathrm{s}) \mathrm{ds},
$$

where $P(s)$ and $M(s)$ are calculated by integrating Equations D3-D5, starting at $t *$, with the initial conditions given by Equations D 8-D10. The probability $\mathrm{P}_{\mathrm{x}}(\mathrm{t} *)$ that at least one transconjugant will appear, then, in a selective sweep beginning at time $t^{*}$, is given by

$$
P_{x}\left(t^{*}\right)=1-e^{-x(t *)} \text {. }
$$

Substituting from (D2) and (D12) into (D1), we have

$$
\begin{aligned}
P_{R} & =\int_{0}^{\infty} \sigma e^{-\sigma t}\left[1-e^{-x(t)}\right] d t \\
& =\int_{0}^{\infty} \sigma e^{-\sigma t}\left[1-e^{-\gamma \iint_{t}^{\rho} P(s) M(s) d s}\right] d t .
\end{aligned}
$$

Finally, to calculate $T_{p}$ from Equation 11, we need expressions for $T_{a}$ and $T_{f}$. Assuming that in a population fixed for the plasmid-borne version of the gene, chromosomals appear as a Poisson process with rate $\chi, T_{a}=$ $1 / \chi . T_{f}$ can be approximated by assuming that the chromosome fixes because no selective sweep occurs; in this case, $T_{f}$ is simply the time required for the number of chromosomals to go from 1 to $>\mathrm{N}-1$, or equivalently, for the frequency of plasmid-bearers to go from 1 $1 / N$ to $<1 / N$. This can be calculated from (D7) by setting $p\left(T_{f}\right)=1 / N$ in (D7) and solving for $T_{f}$. This yields

$$
\mathrm{T}_{\mathrm{f}}=\frac{2 \ln (\mathrm{NV}-1)}{\Psi \alpha(1+\beta)-\gamma \mathrm{N}} .
$$

A note on computation: (D3-D5) cannot be solved 
analytically (H of bauer and Sigmund 1988). However, it is straightforward to solve them by numerical integration. The frequency of plasmid-bearing cells will always be declining at least as fast as in (D7), since mutants, if theyappear, will only hasten the decline of the plasmid (ignoring transconjugants).
Therefore, it is reasonable to end the integration at $T_{f}$, the time at which the number of plasmid-bearing cells is $<1$. Thus, for computational purposes, $T_{f}$ can safely be used as the upper limit of both integrals (replacing $\infty$ ) in (D13). 